\title{
New algorithm for problems with exponential solutions
}

\author{
A. O. Adesanya, R. O. Onsachi, M. R. Odekunle * \\ Department of Mathematic, Modibbo Adama University of Technology, Yola, \\ Nigeria
}

\begin{abstract}
In this paper, we consider the development of algorithms for the solution of first order initial value problems whose solution exhibits exponential behaviour. Method of interpolation and collocation of basis function to give system of nonlinear equations which is solved for the unknown parameters to give a continuous scheme. The discrete methods recovered from the continuous scheme are implemented in block form. The stability properties of the method is verified and numerical experiments show that our method is efficient in handling these problems.
\end{abstract}

Key words: Exponentially-fitted method, interpolation, collocation, stability properties, continuous method, stiff problems

AMS subject classification: 65L05, 65L06

\section{Introduction}

In this paper, we develop an exponentially fitted two step, hybrid numerical integrator for initial value problems (IVPs) of first order differential equations in the form

$$
y^{\prime}=f(x, y), y\left(x_{0}\right)=y_{0}, a \leq x \leq b
$$

where $y:[a, b] \rightarrow \mathbb{R}^{m}, f:[a, b] \times \mathbb{R} \rightarrow \mathbb{R}^{m}, m \geqq 1$ is continuousely differentiable. The Jacobian arising from (1.1)vary slowly and the eigenvalues have negative real part; moreover the solution is decaying or exhibit a pronuounced exponential behaviour.

Classical general purpose method developed using finite power series basis function cannot produce satisfactory results due to the special nature of the problems. Such problems are found in the modeling of disease outbreak, war, radioactive decay, diffusion process in biology and chemical reactions. Several scholars have developed exponentially fitted methods are the best methods for (1.1) among them are [1-8] .

* Corresponding author

Email address: torlar10@yahoo.com, oziohumat@gmail.com, remoodekunle@gmail.com ( A. O. Adesanya, R. O. Onsachi, M. R. Odekunle ). 
The paper is organize as follows: section 2 discusses mathematical background and specification of the method. Section 3 discusses the stability properties of the developed block method which include convergence and stability region of the developed method. Numerical experiments are shown in section 4 and we concluded in section 5 .

\section{Methodology}

We consider the approximate solution

$$
y(x)=\sum_{j=0}^{m} a_{j} x^{j}+\sum_{j=1}^{m} b_{j} e^{-x^{j}}
$$

where $\mathrm{a}_{j}$ and $\mathrm{b}^{\prime}{ }_{j} \mathrm{~s}$ are constants to be determined. We seek approximation at an equidistant set of points defined by the the integration interval $a \leq x_{0}<x_{1}<\cdots<x_{N-1}<x_{N} \leq b$. $x_{n}=x_{0}+n h, n=0,1, \cdots, N-1, h=\frac{b-a}{N-1}, N$ is a positive integer

Interpolating and collocating $(2.1)$ at $x_{n+i}, i=0,1, \cdots, k-1$, give

$$
X A=U
$$

where

$$
\begin{aligned}
& A=\left[\begin{array}{lllll}
a_{0} & a_{1} & a_{2} & \cdots & a_{k-1}
\end{array}\right]^{T} \\
& U=\left[\begin{array}{llllllll}
y_{n} & y_{n+1} & \cdots & y_{n+r} & f_{n} & f_{n+1} & \cdots & f_{n+s}
\end{array}\right]^{T} \\
& X=\left[\begin{array}{ccccccccc}
1 & x_{n} & x_{n}^{2} & \cdots & x_{n}^{k-1} & e^{-x_{n}} & e^{-x_{n}^{2}} & \cdots & e^{-x_{n}^{k}} \\
\vdots & \vdots & \vdots & \vdots & \vdots & \vdots & \vdots & & \vdots \\
1 & x_{n+r} & x_{n+r}^{2} & \cdots & x_{n+r}^{k-1} & e^{-x_{n+r}} & e^{-x_{n+r}^{2}} & \cdots & e^{-x_{n+r}^{k}} \\
0 & 1 & 2 x_{n} & \cdots & (k-1) x_{n}^{k-2} & -e^{-x_{n}} & -2 x_{n} e^{-x_{n}^{2}} & \cdots & -k x_{n} e^{-x_{n}^{k}} \\
\vdots & \vdots & \vdots & \vdots & \vdots & \vdots & \vdots & & \vdots \\
0 & 1 & 2 x_{n+s} & \cdots & (k-1) x_{n+s}^{k-2} & -e^{-x_{n+s}} & -2 x_{n+s} e^{-x_{n+s}^{2}} & \cdots & -k x_{n+s} e^{-x_{n+s}^{k}}
\end{array}\right]
\end{aligned}
$$

$k=r+s-1$. We then impose the following condition on $y(x)$ in $(2.1)$

$$
y\left(x_{n+i}\right)=y_{n+i}, i=0,1 \cdots, r, y^{\prime}\left(x_{n+i}\right)=f_{n+i}, i=0,1, \cdots, s
$$

where $r$ and $s$ are the numbers of interpolation and collocation points respectively. Solving (2.2)using Crammer's rule, substituting the result into (2.1) and after some algebraic simplifications gives the continuous method which when evaluated at selected grid points gives discrete methods.

In this paper, we consider interpolation at $x=x_{n}$ and collocation at $x=x_{n+i}, i=0, \frac{1}{2}, 1,2$. Solving the resulting systems of equation gives the continuous method

$$
y_{n+t}=y_{n}+\gamma_{0} f_{n}+\gamma_{1} f_{n+\frac{1}{2}}+\gamma_{2} f_{n+1}+\gamma_{3} f_{n+2}
$$




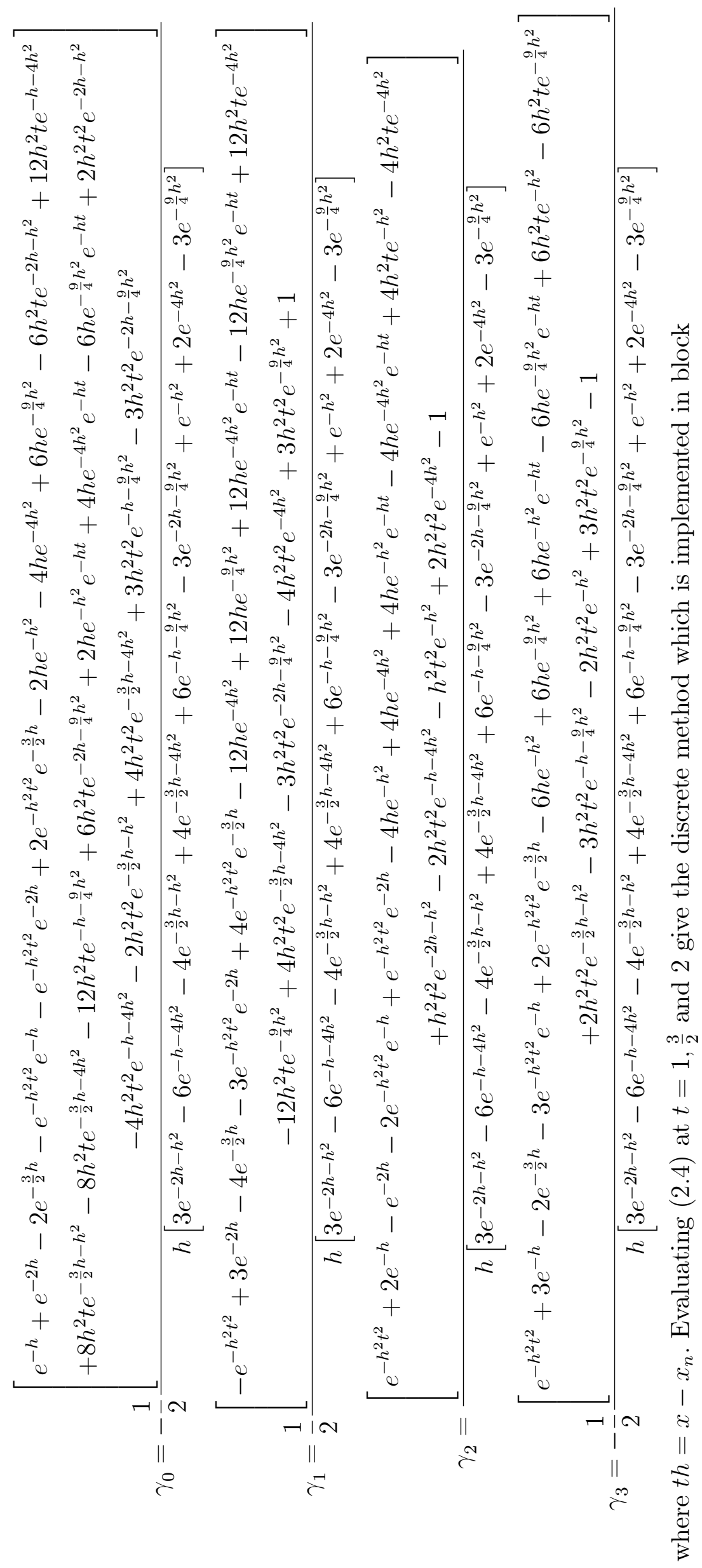




\section{$3 \quad$ Stability properties}

Definition 3.1 If we associate the linear operator $\ell(y(x): h)$ with the method $(2.4)$ defined by

$$
\ell(y(x): h)=y_{n+t}-y_{n}-\left(\gamma_{0} f_{n}+\gamma_{1} f_{n+1}+\gamma_{2} f_{n+\frac{3}{2}}+\gamma_{3} f_{n+2}\right)
$$

where $y(x)$ is continuously differentiable of $[a . b]$. Writing (3.1) as a Taylor series expansion about $x$ to obtain

$$
\ell(y(x): h)=c_{0} y(x)+c_{1} h y^{\prime}(x)+\cdots+c_{p} h^{p} y\left({ }^{p}\right)(x)+\cdots
$$

where the constant coefficient $c_{p}, p=0,1 . \cdots$ are given as

$$
\ell(y(x): h)=\frac{1}{p !}\left(\sum j^{p} \phi_{j}-\frac{1}{(p-1) !} \sum j^{p-1} \psi_{j}-\frac{1}{(p-1) !} \sum p^{j-1} \xi_{j}\right)
$$

then, (3.1)has order $p$ if

$$
\ell(y(x): h)=O\left(h^{p+1}\right), c_{0}=c_{1}=\cdots c_{p}=0, c_{p+1} \neq 0
$$

$c_{p+1}$ is the error constant and the local truncation error $(L T E)=c_{p+1} h^{p+1} y^{(p+1)}$

Table 1: Order and LTE

\begin{tabular}{|c|c|c|}
\hline$t$ & Order & LTE \\
\hline 1 & 4 & $\frac{2}{15} h^{5} \frac{159 y_{n}^{\prime \prime \prime}-4 y_{n}^{5}}{-10795 h^{6}-5040 h^{5}+9856 h^{4}+3168 h^{3}-7168 h^{2}+1536}$ \\
\hline$\frac{3}{2}$ & 4 & $\frac{2}{15} h^{5} \frac{52 y_{n}^{\prime \prime \prime}-\frac{53}{4} y_{n}^{5}}{-10795 h^{6}-5040 h^{5}+9856 h^{4}+3168 h^{3}-7168 h^{2}+1536}$ \\
\hline 2 & 4 & $\frac{2}{15} h^{5} \frac{748 y_{n}^{\prime \prime \prime}-128 y_{n}^{5}}{-10795 h^{6}-5040 h^{5}+9856 h^{4}+3168 h^{3}-7168 h^{2}+1536}$ \\
\hline
\end{tabular}

Definition 3.2 Method (2.4) is consistent if: (i) $\lim _{h \rightarrow 0} \frac{y_{n+t}-y_{n}}{h}=t y_{n}^{\prime}$ (ii) it has order $(p) \geq 1$

Definition 3.3 A method is said to be zero stable if $\lim _{h \rightarrow 0}\left(y_{n+t}\right) \rightarrow y_{n}$

Definition 3.4 A Method is said to be convergent if it is consistent and zero stable.

Definition 3.5 The Region of absolute stability (RAS) of a method is the set

$\{\lambda h:$ for that $\lambda h$ where the root of the stability polynomial are absolute less than one $\}$

In this paper, the boundary locus method is used to plot RAS. This is done by substituting the test equation $y^{\prime}=\lambda y$ into the equation to obtain

$$
\lambda h=z=\frac{\rho(r)}{\sigma(r)}
$$

where $\rho(r)$ and $\sigma(r)$ are the first and second characteristic polynomials respectively. writing $r=e^{i \theta}$ gives RAS as 


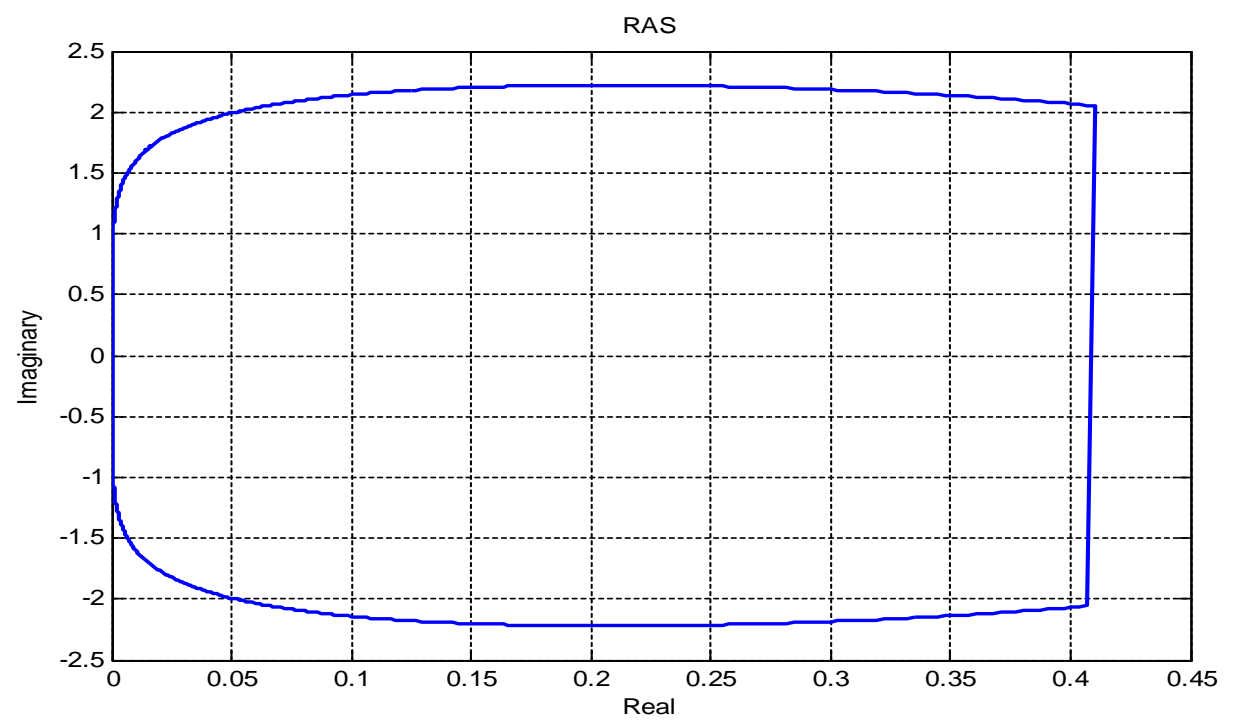

Fig 1: Region of absolute stability of the method

\section{Numerical examples}

The efficiency of the developed method is tested using five test problems with $n=300$

Problem 4.1 We consider the linear system in the range $0 \leq x \leq 1$

$$
y^{\prime}=\left(\begin{array}{cc}
-1 & 95 \\
-1 & -97
\end{array}\right) y, y(0)=\left(\begin{array}{l}
1 \\
1
\end{array}\right)
$$

Source: [2]. The eigenvalues of the Jacobian matrix are $\lambda_{1}=-2, \lambda_{2}=-96$ with the stiffness ratio 48 . The exact solution is given as $y(x)=\frac{1}{47}\left(95 e^{-2 x}-48 e^{-95 x}, 48 e^{-96 x}-e^{-2 x}\right)^{T}$. The results is shown in Table II 


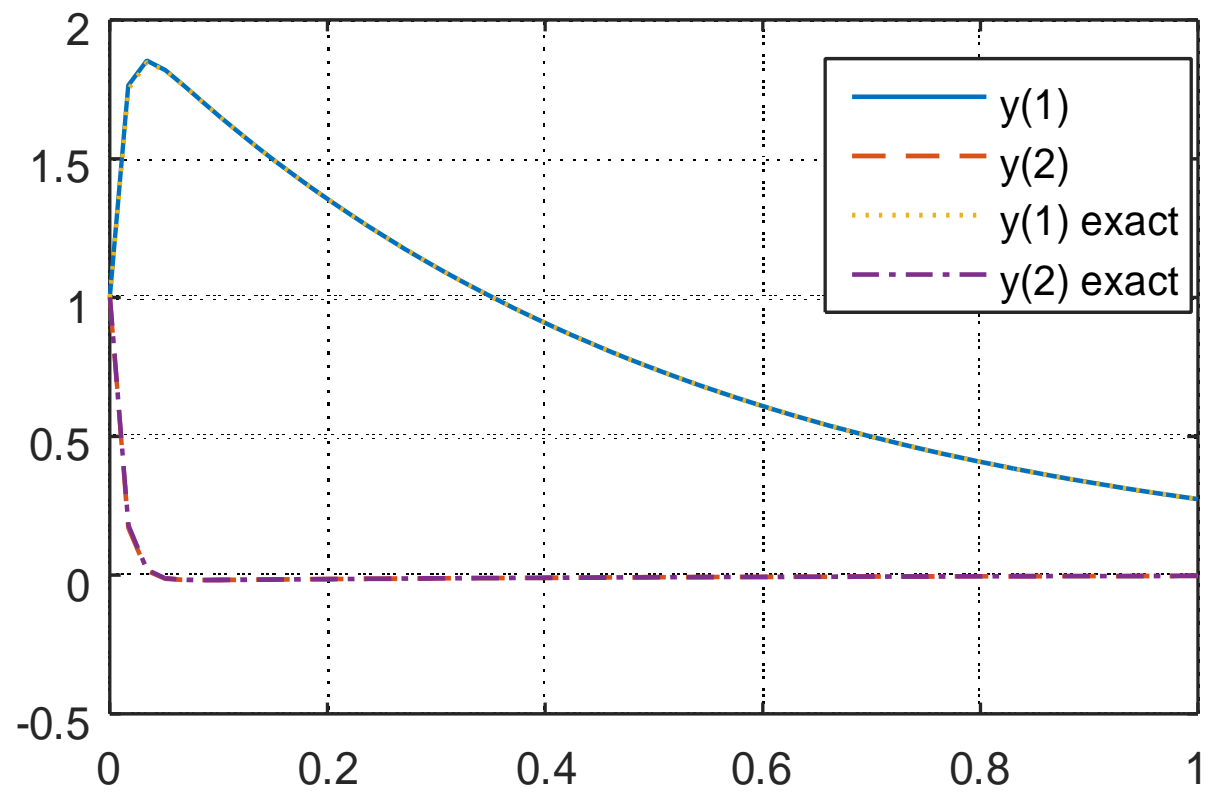

Fig. 2: Results of problem 4.1

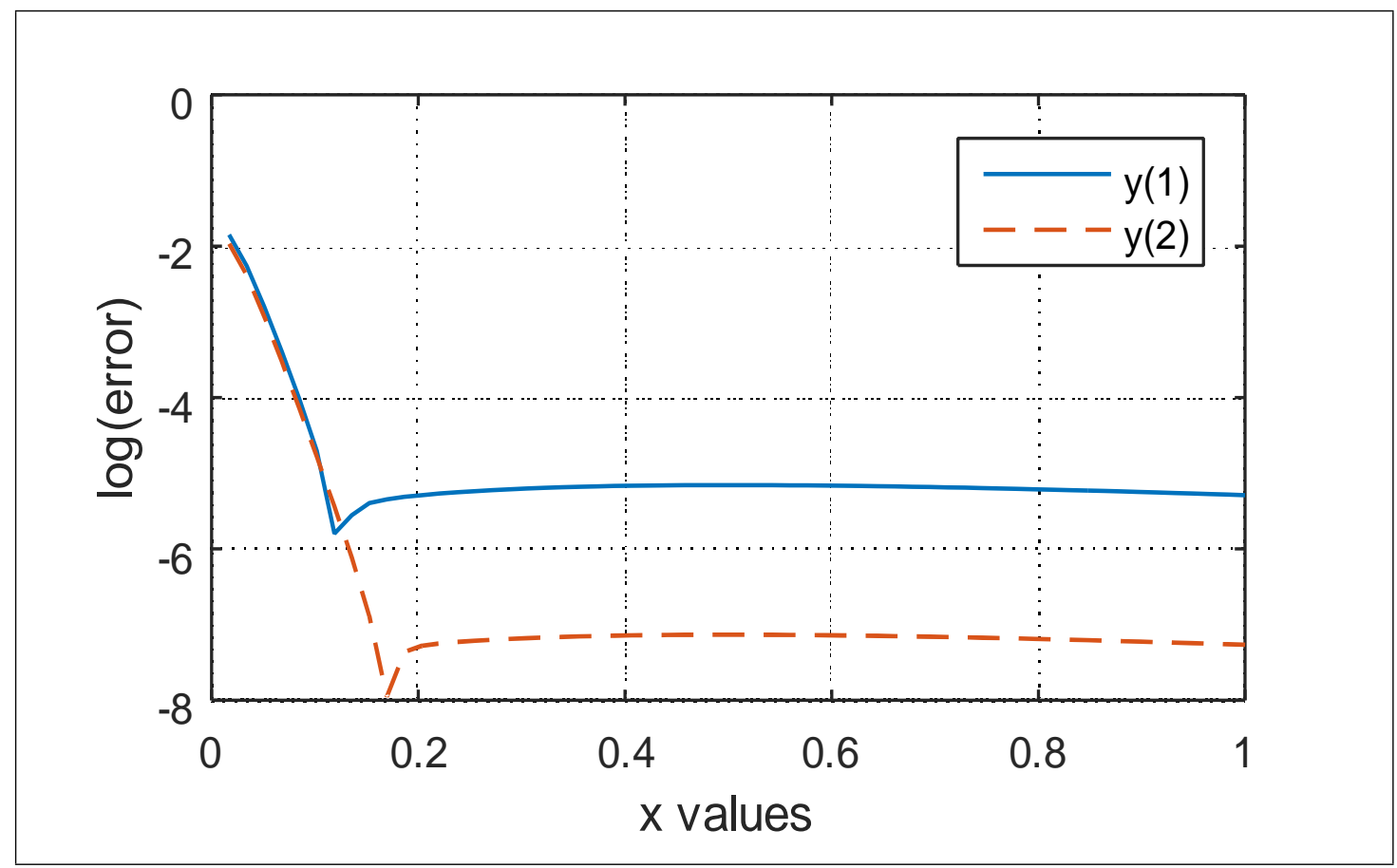

Fig. 3: $\log ($ error) against $\mathrm{x}$ values for problem 4.1

Problem 4.2 We consider a non-linear system in the range $0 \leq x \leq 20$

$$
\left(\begin{array}{l}
y_{1}^{\prime}(x) \\
y_{2}^{\prime}(x)
\end{array}\right)=\left(\begin{array}{c}
-1002 y_{1}(x)+1000 y_{2}^{2}(x) \\
y_{1}(x)-y_{2}(x)\left(1+y_{2}(x)\right)
\end{array}\right),\left(\begin{array}{l}
y_{1}(0) \\
y_{2}(0)
\end{array}\right)=\left(\begin{array}{l}
1 \\
1
\end{array}\right)
$$

with exact solution $(y(x))=(\exp (-2 x), \exp (-x))^{T}$.

Source: [10] 


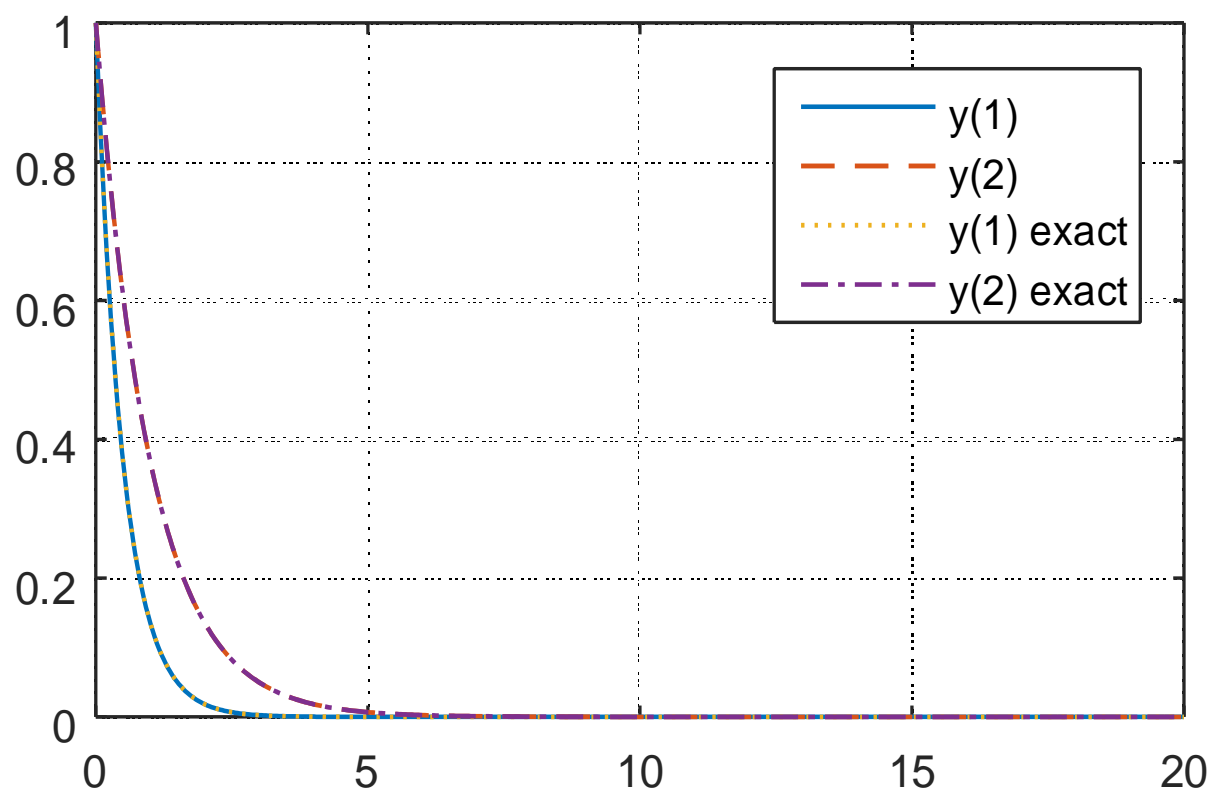

Fig. 4: Results of problem 4.2

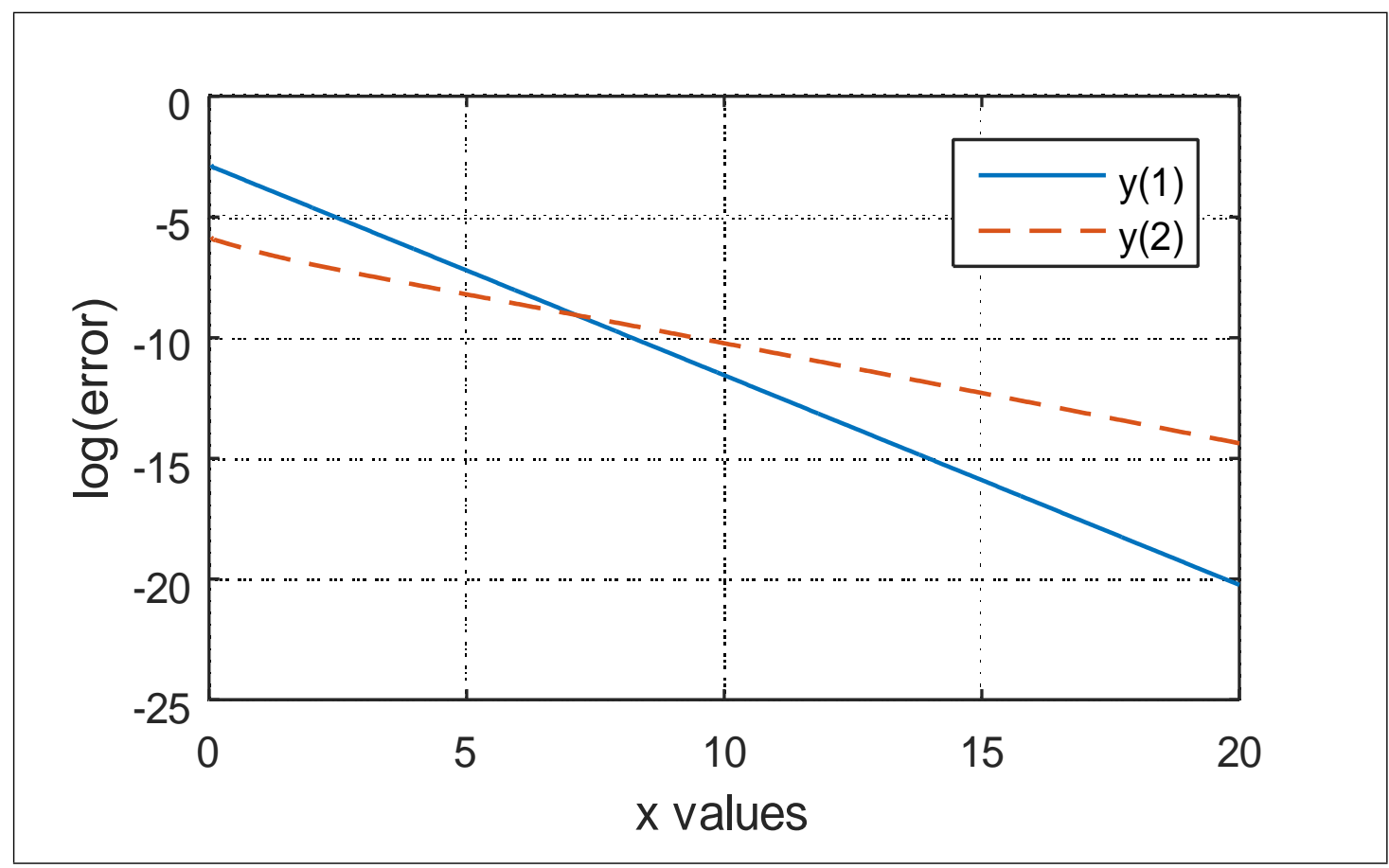

Fig. 5: $\log ($ error) against $\mathrm{x}$ values for problem 4.2

Problem 4.3 Consider a system in the range $0 \leq t \leq 10$

$$
y^{\prime}=\left(\begin{array}{cc}
-1 & -30 \\
30 & -1
\end{array}\right) y+\left(\begin{array}{c}
30 e^{-t} \\
-30 e^{-t}
\end{array}\right) y, y(0)=\left(\begin{array}{l}
1 \\
1
\end{array}\right)
$$

with exact solution $y(x)=\left(e^{-t}, e^{-t}\right)^{T}$

Source:[4] 


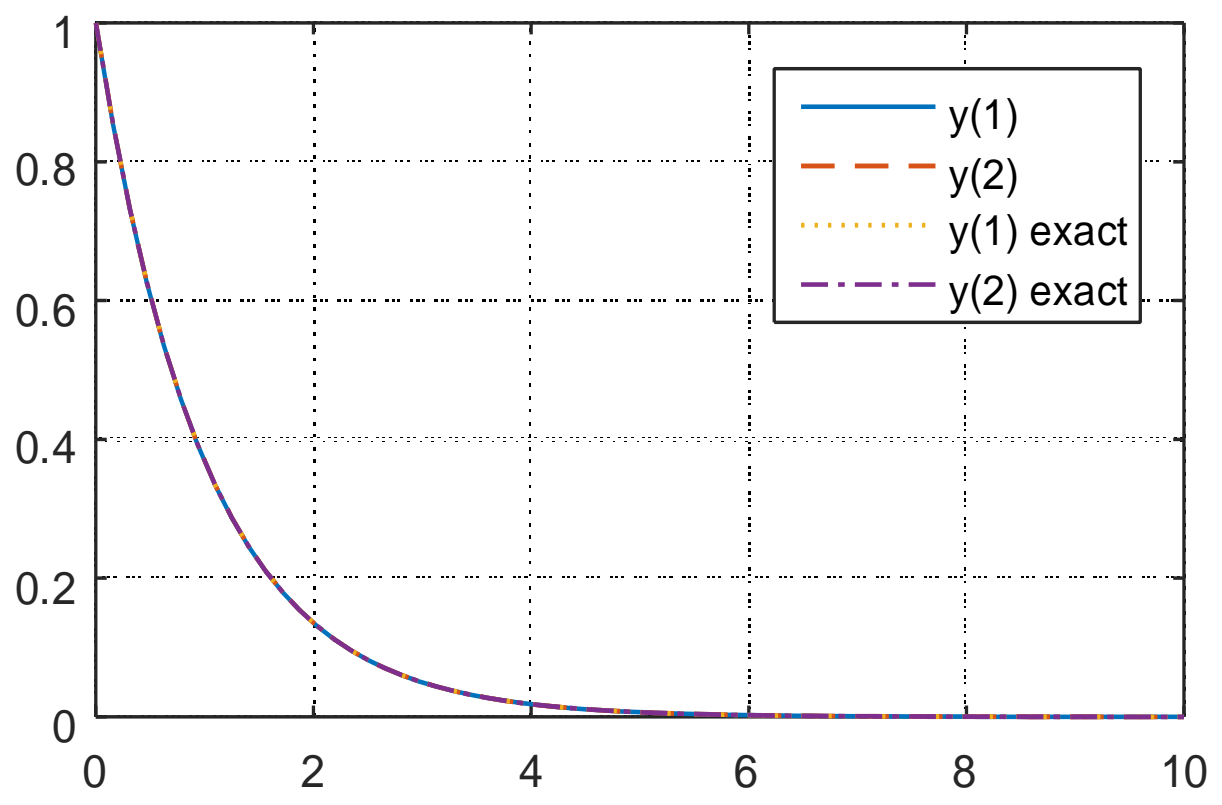

Fig. 6: Results of problem 4.3

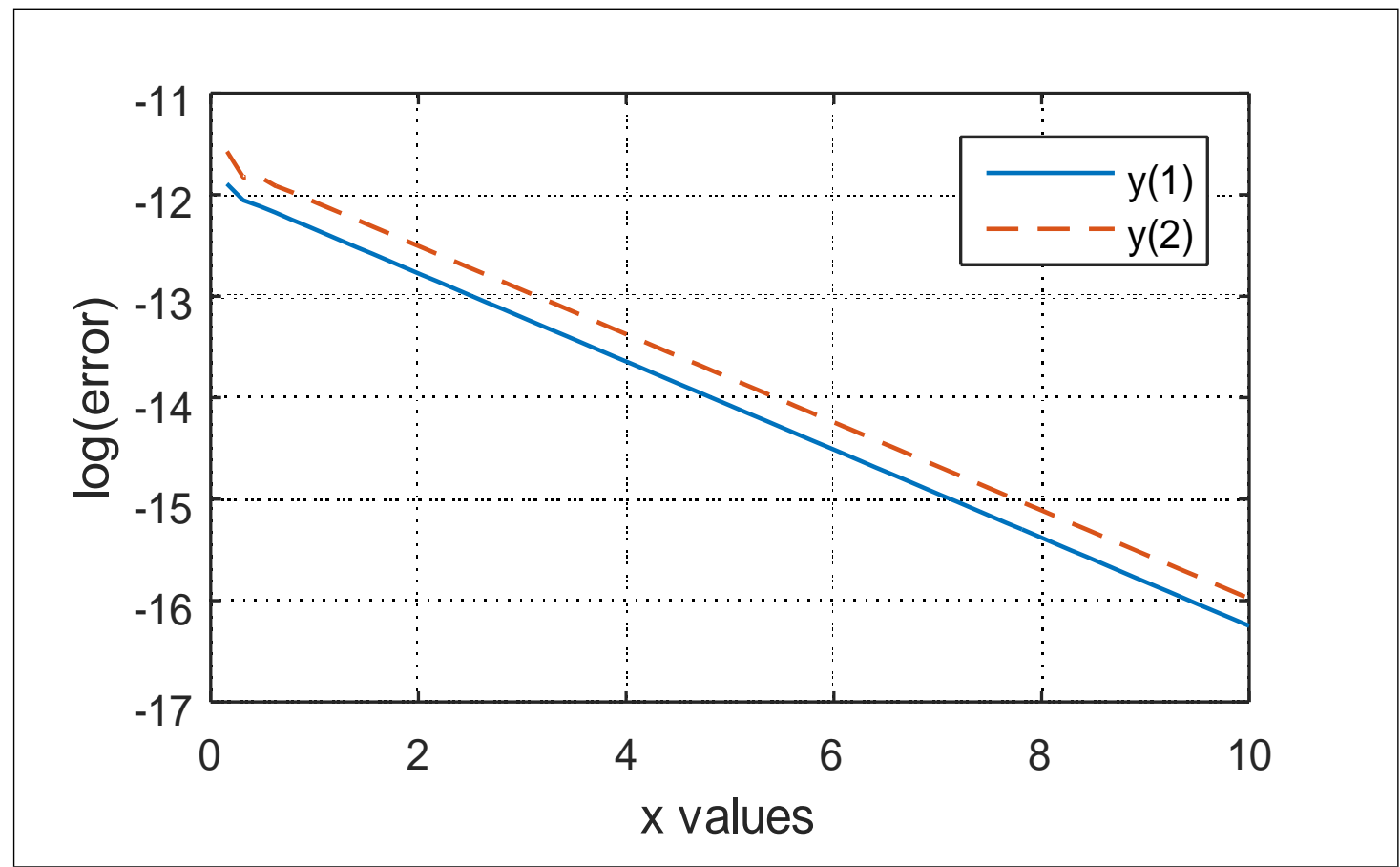

Fig. 7: $\log ($ error) against $\mathrm{x}$ values for problem 4.3

Problem 4.4 consider the stiff system in the interval $0 \leq t \leq 5$

$$
\left(\begin{array}{l}
y_{1}^{\prime} \\
y_{2}^{\prime}
\end{array}\right)=\left(\begin{array}{l}
-1001 y_{1}+999 y_{2}+2 y_{1} y_{2} \\
999 y_{1}-1001 y_{2}+y_{1}^{2}+y_{2}^{2}
\end{array}\right),\left(\begin{array}{l}
y_{1}(0) \\
y_{2}(0)
\end{array}\right)=\left(\begin{array}{c}
0 \\
-1
\end{array}\right)
$$

with the exact solution $y(x)=\left(\frac{1000}{2001 e^{2000 t}-1}-\frac{1}{3 e^{2 t}-1},-\frac{1000}{2001 e^{2000 t}-1}-\frac{1}{3 e^{2 t}-1}\right)^{T}$.

Source: [8] 


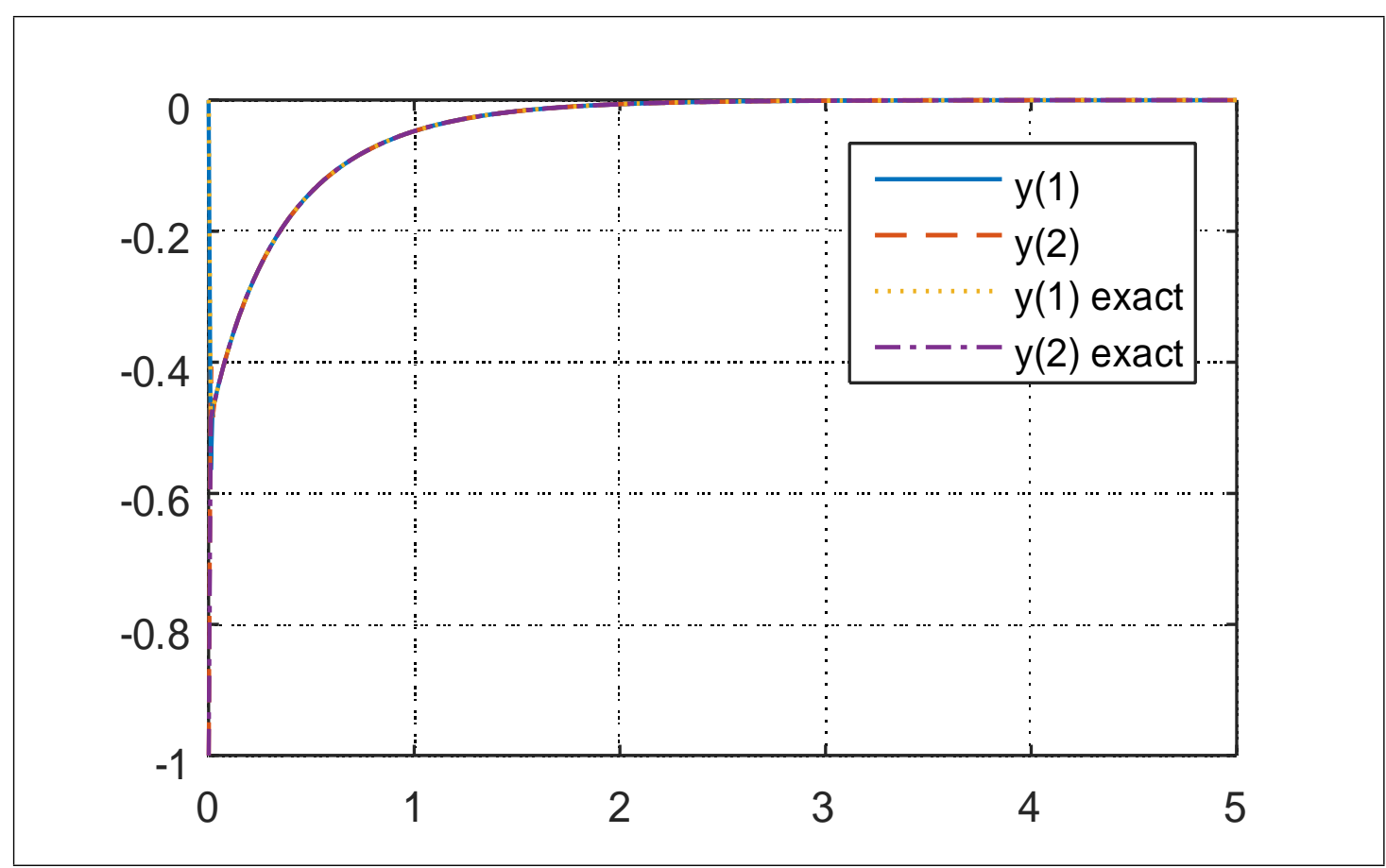

Fig. 8: Results of problem 4.4

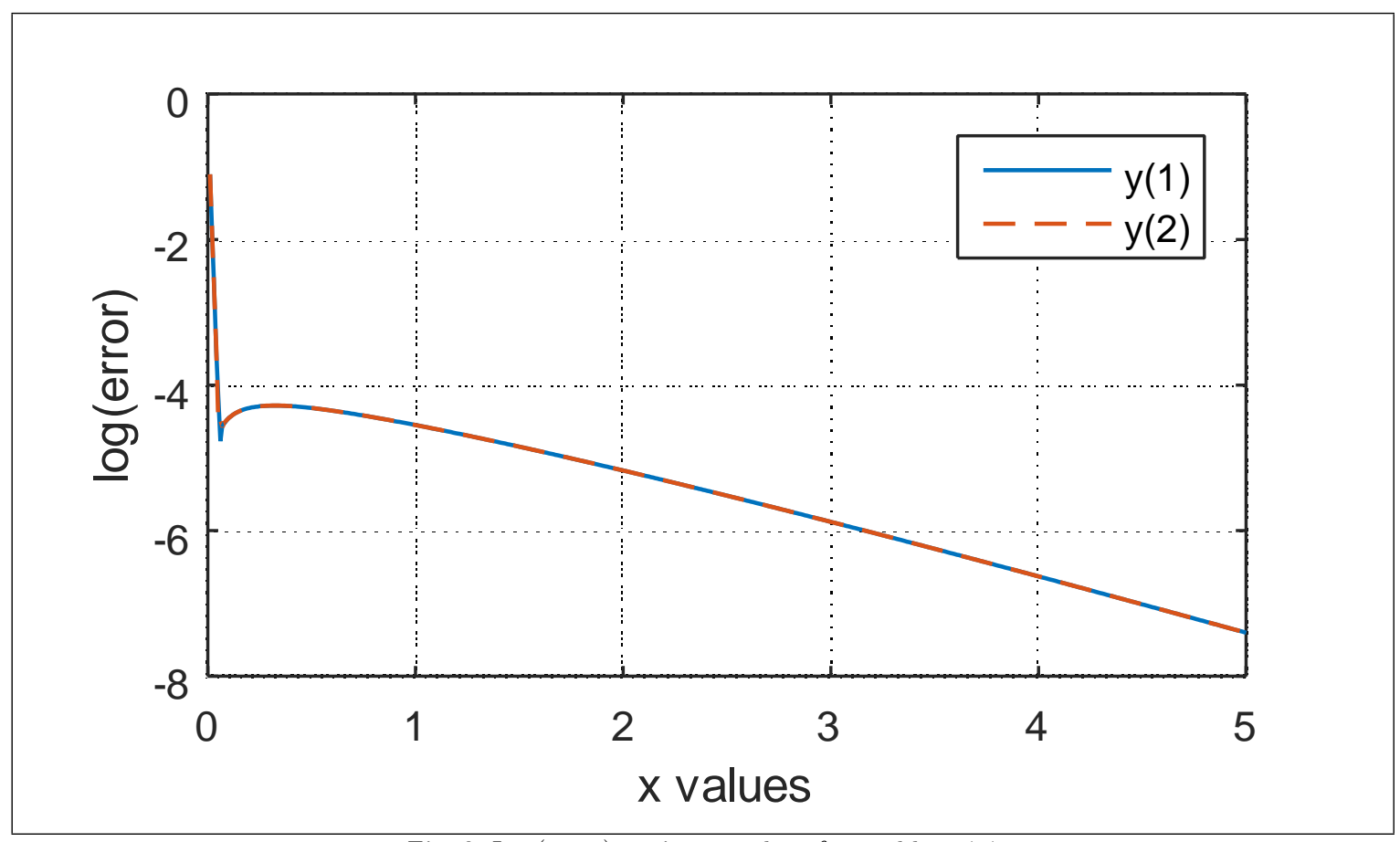

Fig. 9: $\log$ (error) against $\mathrm{x}$ values for problem 4.4

Problem 4.5 consider the simple nonlinear stiff problem within the interval $x=[0,20]$

$$
\left(\begin{array}{l}
y_{1}^{\prime} \\
y_{2}^{\prime}
\end{array}\right)=\left(\begin{array}{c}
-y_{1} \\
2 y_{1}^{2}-2 y_{2}
\end{array}\right),\left(\begin{array}{l}
y_{1}(0) \\
y_{2}(0)
\end{array}\right)=\left(\begin{array}{l}
5 \\
5
\end{array}\right)
$$

with the exact solution $y(x)=\left(5 e^{-x}, 5 e^{-2 x}(1+5 x)\right)^{T}$.

Source: $[6]$ 


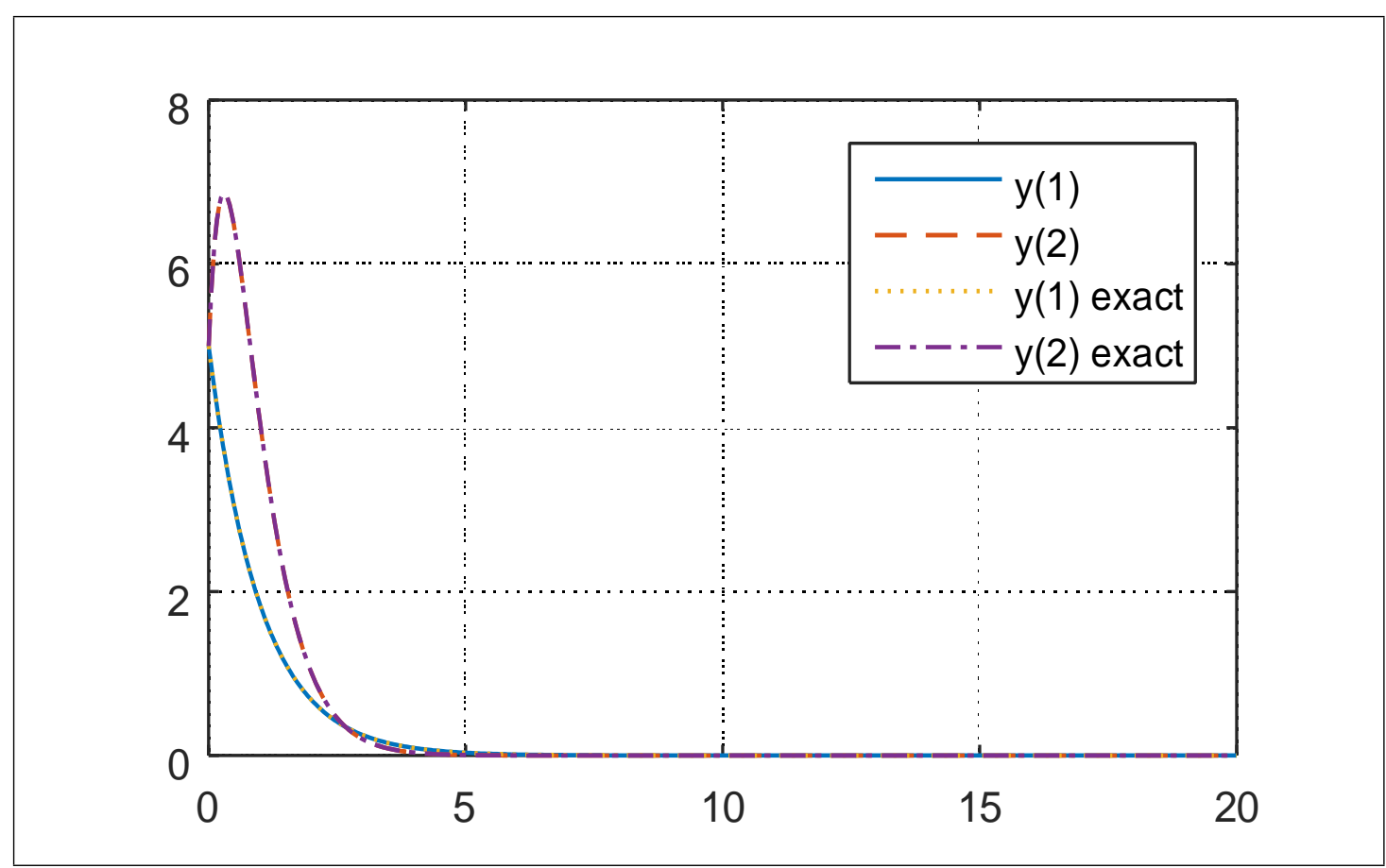

Fig. 10: Results of problem 4.5

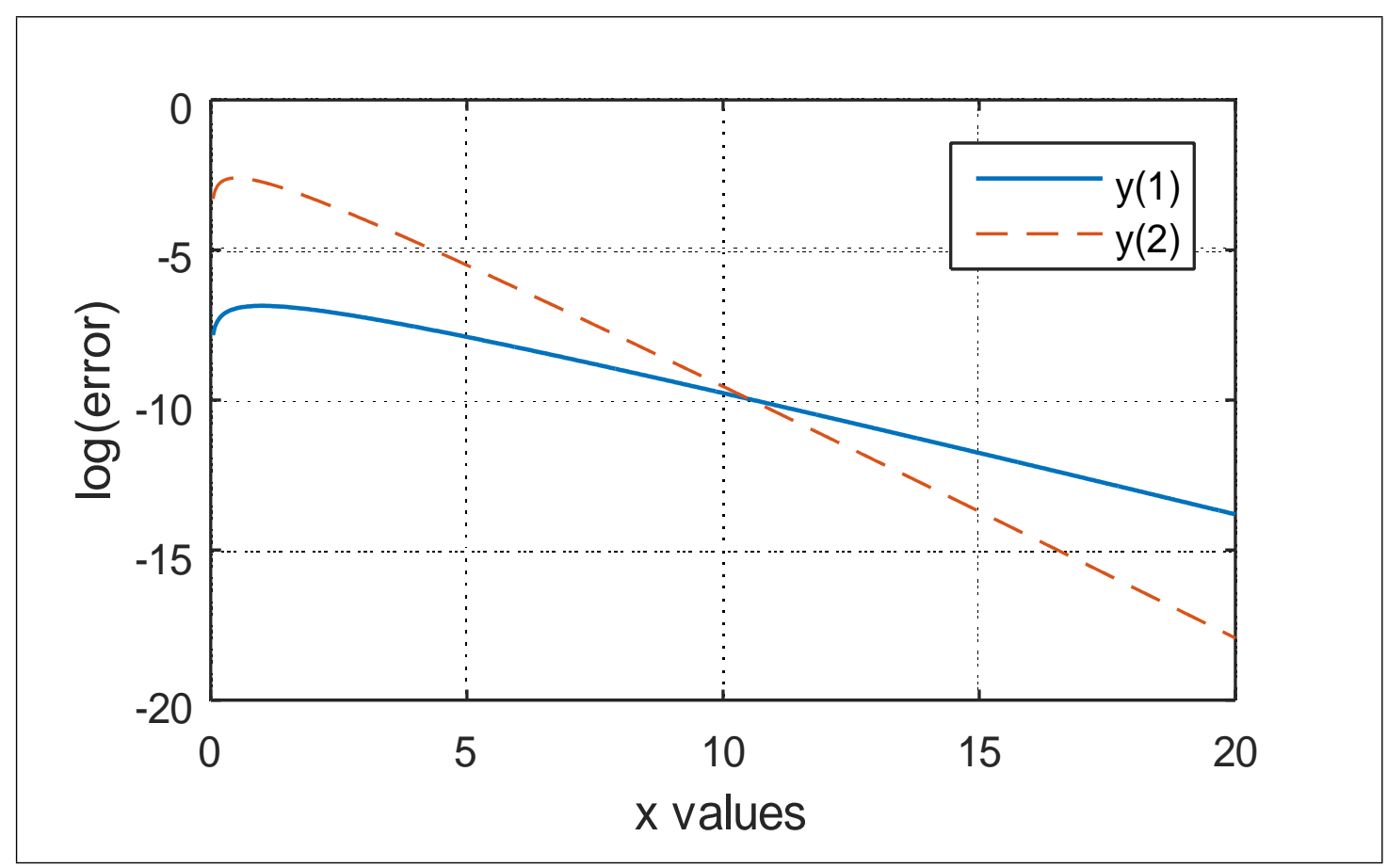

Fig. 10: $\log ($ error) against $\mathrm{x}$ values for problem 4.5

\section{Conclusion}

We have discussed the construction of order four hybrid method for stiff first order IVPs. The method is found suitable for problems whose solution exhibit exponential behaviour or decaying as demostrated in the numerical experiments. 


\section{References}

[1] G. V. Berghe, H. D. Meyer, M. V. Daele, T. V. Hecke. Exponentially fitted Runge Kutta method. Journal of Computational and Applied Mathematics, 125(2000),107-115

[2] C. E. Abdulimen. Exponentially fitted third derivative three step method for numerical integration of stiff initial value problems. Applied Mathematics and Computation, 243, (2014), 446-453

[3] Y. Fengjian, C. Xinming, L. Yiping. High order one step A-stable exponentially fitted method. Appl. Math. J. Chiness Univ. Ser. B. 14(3), (1999), 357-365

[4] T. E. Simon. Exponentially-fitted Runge Kutta- Nystrom method for the numerical solution of initial value problems with oscillating solutions. Applied Mathematics Letters. 15, (2002), $217-225$

[5] T. Y. Ying, N. Yaacob. One step exponentially-rational method for the numerical solution of first order initial value problems. SIAM Malaysiana, 42(6), (2013), 845-853

[6] J. Carroll. A matricial exponentially fitted scheme for the numerical solution of stiff initial value problems. computers. Math. Applic. 26(4), (1993), 57-64

[7] Y. Yang, Y. Fang, X. You, B. Nang. Novel exponentially fitted two derivative Runge Kutta methods with equation-dependent coefficient for first order differential equations. Discrete Dynamics in Nature and Society. doi.org/10.1152/2016/9827952

[8] A. Xiao, G. Zhang, X. Yi. Implicit-explicit multistep method for nonlinear stiff initial value problems. applied Mathematics and Computations, 247, (2014), 47-60

[9] S. O. Fatunla. Nonlinear multistep methods for initail value problems. Comp. \& Maths. with Appls. 8(3), (1993) 231-239

[10] D. G Yakubu, S. Markus. The efficiency of second derivative multistep methods for the numerical integration of stiff systems. Journal of the Nigerian Mathematical Society, (2016). doi.org/10.1016/j.jnnms.2016.02.002 